Sharif University of Technology
Scientia Iranica
Transactions E: Industrial Engineering
wCIENTIA

\title{
Bi-level programming for supplier selection under quantity discount policy
}

\author{
F. Zaheri ${ }^{\mathrm{a}, *}$, M. Zandieh ${ }^{\mathrm{b}}$ and M.T. Taghavifard ${ }^{\mathrm{c}}$ \\ a. Department of Industrial Engineering, Science and Research Branch, Islamic Azad University, Tehran, Iran. \\ b. Department of Industrial Management, Faculty of Management and Accounting, Shahid Beheshti University, G.C., Tehran, Iran. \\ c. College of Management and Accounting, Allameh Tabataba'i University, Tehran, Iran.
}

Received 9 September 2015; received in revised form 16 May 2016; accepted 13 August 2016

\section{KEYWORDS}

Supply chain;

Bi-level programming;

Supplier selection;

PSO.

\begin{abstract}
This paper proposes two models to formulate a Supplier Selection Problem (SSP) in a single-buyer, multi-supplier two-echelon supply chain network. The model coordinates order allocation and supplier selection problems under all-unit quantity discount policy. In this way, bi-level programming is employed to obtain two models: 1) The model with buyer as a leader; 2) The model with vendor as a leader. The resulted nonlinear bi-level programming problems are hard to solve. Therefore, Particle Swarm Optimization (PSO) algorithm is used to deal with the complexity of the model and makes it solvable. Numerical results show that the proposed model is efficient for SSP in compliance with order allocation decision making.
\end{abstract}

(C) 2017 Sharif University of Technology. All rights reserved.

\section{Introduction}

A Supply Chain (SC) is a complex hierarchical system, including several contributors, i.e. suppliers, manufacturers, distributors, retailers, and ultimate consumers [1]. Each contributor, in this network, tries to control a set of decision variables and makes decisions based on its needs and interests. Therefore, some studies have studied the SC components cooperation based on pricing, inventory and production planning, supplier selection, product designation, capacity allocation, and so on [2].

This paper considers a network with a single vendor and multiple suppliers to feed the final customer. Since the final model is based on two distinct levels, it is not suitable to use the standard programming to formulate problems [3]. Therefore, in this research, Bi-Level Programming (BLP) approach is used to for-

*. Corresponding author. Tel./Fax: +98 2147911 E-mail addresses: Fzaheri2002@gmail.com (F. Zaheri); m_zandieh@sbu.ac.ir (M. Zandieh); taghavifard@atu.ac.ir (M.T. Taghavifard). mulate a Supplier Selection Problem (SSP) in Supply Chain Network (SCN).

BLP is a proper model to deal with noncooperative decision-makers in decision-making processes [3]. The aim of the BLP is to formulate problems with hierarchical structure and two levels of decisionmaking [4]. The leader, at a higher level of hierarchy, follows a specific strategy, and the follower, at a lower level of hierarchy, determines its strategy subsequently. In such a non-cooperative game, each decision-maker controls a set of decision variables and tries to optimize his/her own objective function [5]. Stackelberg reported the follower as the dominated member of the game which is controlled by the leader. Given the estimation of the followers' reaction, the leader takes the first step [6]. According to its characteristics, the BLP approach has been applied in so many fields, i.e. environmental economic problem [7], optimal design engineering [8], mechanical engineering, decentralized resource planning, logistics and transportation [9], civil engineering, electric power markets [10], and so on.

More generally, the BLP can be formulated in 


$$
\begin{aligned}
& \text { Eq. (1): } \\
& \min _{x, y} f_{1}(x, y) \\
& \text { s.t. }(x, y) \in R,
\end{aligned}
$$

where $y$ is a solution vector for any given $x$ obtained by Eq. (2):

$$
\begin{aligned}
& \min _{y} f_{2}(x, y), \\
& \text { s.t. }(x, y) \in S .
\end{aligned}
$$

On the other hand, $x \in R^{n_{1}}$ is the vector of variables, controlled by the leader, and $y$ is the vector of variables, controlled by the follower.

In spite of all its useful characteristics, it is really difficult to solve the BLP problems. Even with the problems that objective function and all constraints are linear, these problems are considered as NP-hard [11]. Therefore, several different methods have been proposed to obtain exact solution to the BLP issues and its applications, i.e. enumerative method [12], using the penalty function [13], and a method based on the Gradient method [5]. Some of approaches and algorithms, introduced to solve linear BLP problems, are based on Karush-Kunh-Tucker conditions [14], branch-and-bound algorithm [11], and Benders decomposition algorithm [15]. Since it is difficult to solve the BLP problems, meta-heuristic approaches have expanded. Meta-heuristic approaches have greater ability to overcome many challenges of the BLP problems. Genetic algorithm [16], ant colony optimization algorithm [5], differential evolution [17], Particle Swarm Optimization (PSO) [10], and coevolutionary approaches [18] are some meta-heuristic approaches introduced to solve the BLP problems.

$\mathrm{Yu}$ et al. [19] proposed the BLP for a SC with Vendor Managed Inventory (VMI) strategy. In their model, a manufacturer plays the role of a leader and several retailers play the role of followers. Both manufacturers and retailers try to obtain their own optimal product marketing and inventory policies. Kis and Kovács [20] determined incapacitated lot-sizing strategy in compliance with backlogs in a two-echelon SC using BLP approach. Sadigh et al. [21] introduced a framework for a multi-product $\mathrm{SC}$ with a single manufacturer and single retailer using Stackelberg game theory. In their model, the demand is a nonlinear function of prices and advertising expenditures. Wee et al. [22] considered a vendor as a leader and a buyer as a follower to formulate a SC through BLP problem approach. They captured a time-varying price and solved the model with bi-level elitist genetic algorithm.

Supplier selection is a key issue in decision making process involving many respects, i.e. quality and reliability, along with measures such as discounts and pricing [23]. There are a lot of studies prominent in the field of supplier selection. Rosenblatt et al. [24] studied procurement policies and order quantity in a SC. Xia and Wu [25] considered quantity discounts in a SSP. They referred to the issues of multiple suppliers to provide multiple products with capacity limits, price intervals, and quantitative discounts. J.L. Zhang and M.Y. Zhang [26] studied the same problem for a single manufacturer with a random demand in which suppliers have different sales prices and limited capacities. They also considered holding, shortage, and fixed order costs. Mohamadi et al. [27] introduced an integrated model for supplier location selection and order allocation with capacity constraints under uncertainty. They formulated a bi-objective model to optimize SCN costs. Neyestani et al. [28] investigated a supplier order allocation with one buyer and preselected suppliers. They formulated a multi-objective mixed integer nonlinear programming problem to optimize the total value and cost of purchase.

To the best of our knowledge, researchers try to formulate SSP using integrated mathematical programming. Nevertheless, the problem has a hierarchical nature, and it is better to employ the BLP to formulate it. This research consists of a buyer, a vendor, and multiple suppliers. The BLP approach is proposed for supplier selection and order allocation under the all-unit quantity discount policy. There are two main questions which are investigated:

1. Is the cost of the integrated model for supplier selection less than the BLP approach?

2. How can an effective approach be developed for solving the resulted nonlinear BLP?

Given the order quantity per period, the paper adopts the BLP approach to formulate the SSP with taking the suppliers' capacity in to account. Since the objective functions of the leader and the follower are both nonlinear, the problem is formulated as a Non-Linear Bi-Level Programming (NLBLP). It determines the amount of each order per period and selects a set of capacitated suppliers. In this case, it is assumed that the buyer and vendor play the role of the leader and the follower in the hierarchical structure of decisionmaking.

The rest of the paper is as follows: the problem statement and formulation will be described in Section 2 with details. The solution approach, computational results, and selectivity analysis will be pointed out in Section 3, and finally conclusions and future studies will be presented in Section 4.

\section{Problem statement}

This paper addresses the SSP, including a buyer and 
a vender with multiple suppliers. The buyer selects one or more suppliers to order a product with a fixed market demand. All capacitated suppliers offer all-unit quantity discounts with predetermined discount intervals and limited annual production rate. Furthermore, the economic quantity of production is equivalent to that of purchase order, which is requested by the buyer.

\subsection{Model assumptions and notations}

The following assumptions are considered in the model formulation:

- The buyer can provide the required quantity of supply from multiple suppliers;

- There is only one product specified in this chain;

- The vendor offers all-unit quantity discount and discounted prices will be applied to the orders;

- Specific annual demand is known and it has a constant amount over time;

- Inventory shortage is not allowed for both buyer and the suppliers;

- Inventory is not transferable from one period to another period.

The parameters and decision variables used in the model formulation are as follows:

\section{The parameters:}

$i \quad$ Index representing the suppliers of the vendor

$k \quad$ Index for discount intervals

$n \quad$ The number of suppliers

$\xi \quad$ A small positive number

$l_{i k} \quad$ The lower bound of discount interval $k$ offered by supplier $i$

$u_{i k} \quad$ The upper bound of discount interval $k$ offered by supplier $i$

$c_{i k} \quad$ The discounted unit price in discount interval $k$ offered by supplier $i$

$K_{i} \quad$ The index of the last discount interval offered by supplier $i$

$D \quad$ Annual demand rate for the buyer

$A_{i} \quad$ The cost of each order from supplier $i$

$S_{i} \quad$ The setup cost of production for supplier $i$

$P_{i} \quad$ Annual production rate for supplier $i$

$z_{i} \quad$ Variable cost per unit of product for suppliers $i$

$h_{b} \quad$ Buyers' inventory holding cost per unit per unit time

$h_{i} \quad$ Suppliers' $i$ holding cost per unit per unit time
The decision variables:

$q_{i k} \quad$ The quantity purchased in each period from a supplier $i$ in discount interval $k$

$y_{i k} \quad$ Binary variable; if purchased quantity provided from suppliers $i$ per period be placed in discount interval $k$, value $y_{i k}=1$; otherwise, $y_{i k}=0$

$Q \quad$ The total value of the order per period for the buyer from all suppliers

$y_{i} \quad$ Binary variable; if supplier $i$ has been chosen by the buyer, value $y_{i}=1$; otherwise, $y_{i}=0$

\subsection{BLP model for SSP: Buyer as a leader}

The BLP formulation of the model is described through Eqs. (3) to (13):

$$
\begin{array}{r}
\min _{Q, y} z_{L}\left(Q, y_{i}\right)=D / Q \sum_{i=1}^{n} \sum_{k=1}^{K_{i}}\left(c_{i k} q_{i k}+A_{i} y_{i}\right) \\
+h_{b} / 2 Q \sum_{i=1}^{n}\left[\left(\sum_{k=1}^{K_{i}} q_{i k}\right)^{2}\right]
\end{array}
$$

s.t.:

$Q \geq \xi$

$\sum_{k=1}^{K_{i}} y_{i k} \leq y_{i} \quad \forall i=1, \cdots, n$,

$y_{i} \in\{0,1\} \quad \forall i=1, \cdots, n$,

$\min _{q, y} z_{F}\left(q_{i k}, y_{i k}\right)=D / Q \sum_{i=1}^{n} \sum_{k=1}^{K_{i}}\left(z_{i} q_{i k}+S_{i} y_{i k}\right)$ $+D / 2 Q \sum_{i=1}^{n}\left[\frac{h_{i}}{P_{i}}\left(\sum_{k=1}^{K_{i}} q_{i k}\right)^{2}\right]$

s.t.:

$\sum_{k=1}^{K_{i}} q_{i k}=Q$

$\sum_{k=1}^{K_{i}} q_{i k} \leq \frac{P_{i}}{D} Q \quad \forall i=1, \cdots, n$,

$y_{i k} l_{i k} \leq q_{i k} \leq u_{i k} y_{i k} \quad \forall i=1, \cdots, n, \forall k=1, \cdots, K_{i}$,

$\sum_{k=1}^{K_{i}} y_{i k} \leq 1 \quad \forall i=1, \cdots, n$,

$y_{i k} \in\{0,1\} \quad \forall i=1, \cdots, n, \quad \forall k=1, \cdots, K_{i}$,

$q_{i k} \geq 0 \quad \forall i=1, \cdots, n, \quad \forall k=1, \cdots, K_{i}$. 
Eqs. (3) and (7) represent higher and lower level objective functions, respectively. Eq. (3) reflects the total annual cost of purchasing, order, and holding for the leader, while Eq. (7) represents the total annual cost of production, setup, and holding for the suppliers. Constraint (4) denotes the fact that the orders received from all suppliers must be greater than zero. Constraint (5) expresses the fact that if a supplier is selected by the buyer, it will be able to offer one of its price intervals. Constraint (6) is to select or unselect any of the suppliers. Constraint (8) embraces the fact that the quantity of each order per period by the buyer equals the total quantity per period received from all selected suppliers of the vendors in each order. Constraint (9) indicates that the annual quantity of the order from a supplier would not exceed the annual production capacity of that supplier. Constraints (10) and (11) enforce that each supplier can feed the network according to its capacity interval. Constraint (12) denotes binary variable, $y_{i k}$, and Constraint (13) indicates that $q_{i k}$ variables are positive.

\subsection{BLP models for SSP: Vendor as a leader}

The BLP formulation of the model is described through Eqs. (14) to (24):

$$
\begin{aligned}
\min _{q, y} z_{L}\left(q_{i k}, y_{i k}\right)= & D / Q \sum_{i=1}^{n} \sum_{k=1}^{K_{i}}\left(z_{i} q_{i k}+S_{i} y_{i k}\right) \\
& +D / 2 Q \sum_{i=1}^{n}\left[\frac{h_{i}}{P_{i}}\left(\sum_{k=1}^{K_{i}} q_{i k}\right)^{2}\right]
\end{aligned}
$$

s.t.:

$$
\begin{array}{ll}
\sum_{i=1}^{n} \sum_{k=1}^{K_{i}} q_{i k}=Q, & \\
\sum_{k=1}^{K_{i}} q_{i k} \leq \frac{P_{i}}{D} Q & \forall i=1, \cdots, n, \\
y_{i k} l_{i k} \leq q_{i k} \leq u_{i k} y_{i k} & \forall i=1, \cdots, n, \\
& \forall k=1, \cdots, K_{i}, \\
\sum_{k=1}^{K_{i}} y_{i k} \leq 1 & \forall i=1, \cdots, n, \\
y_{i k} \in\{0,1\} & \forall i=1, \cdots, n, \\
& \forall k=1, \cdots, K_{i}, \\
q_{i k} \geq 0 & \forall i=1, \cdots, n,
\end{array}
$$

$$
\begin{aligned}
\min _{Q, y} z_{F}\left(Q, y_{i}\right)= & D / Q \sum_{i=1}^{n} \sum_{k=1}^{K_{i}}\left(c_{i k} q_{i k}+A_{i} y_{i}\right) \\
& +h_{b} / 2 Q \sum_{i=1}^{n}\left[\left(\sum_{k=1}^{K_{i}} q_{i k}\right)^{2}\right],
\end{aligned}
$$

s.t.:

$$
\begin{aligned}
& Q \geq \xi, \\
& \sum_{k=1}^{K_{i}} y_{i k} \leq y_{i} \quad \forall i=1, \cdots, n, \\
& y_{i} \in\{0,1\} \quad \forall i=1, \cdots, n .
\end{aligned}
$$

\section{The proposed solution method}

The BLP problem is a NP-hard one due to its hierarchical structure [22]. Although it is easier to model a hierarchical problem by using the BLP approach, employing only exact solution methods is very hard and time-consuming. Therefore, it is better to use other methods to solve such a model. Since both of the formulated models are mixed binary NLBLP, the meta-heuristic algorithm is adopted to solve them based on PSO algorithm. On the other hand, the method needs the follower solution in the procedure. This solution may be obtained using the Branch-AndReduce Optimization Navigator (BARON) as a solver of GAMS software for nonlinear models. In other words, PSO algorithm, as a well-known meta-heuristic method, is employed to deal with the higher level of the problem. Due to nonlinearity of the problem, the BARON solver is employed to obtain a global solution to the problem in its lower level. Some more details are illustrated in Figure 1 and rather discussed as follows.

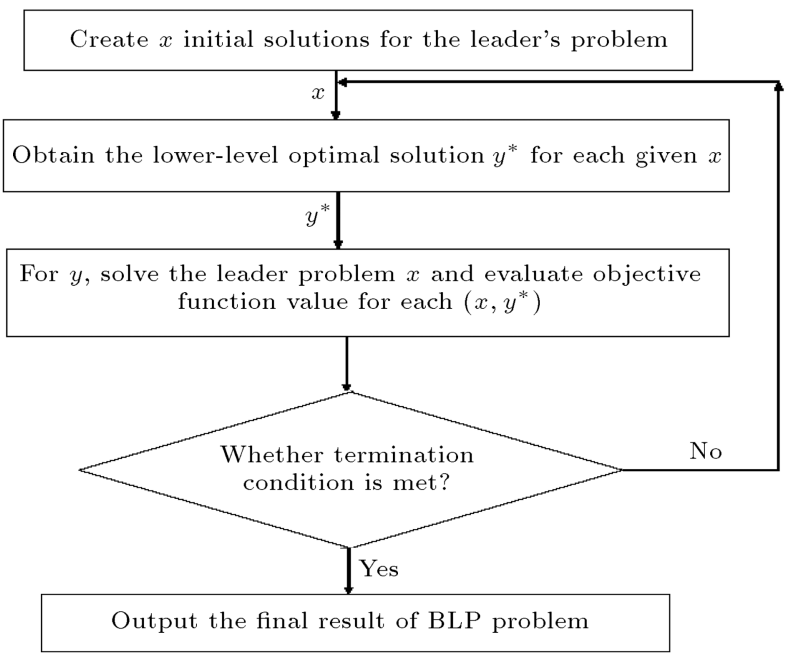

Figure 1. The scheme procedure for solution. 
According to the structure of the BLP at Eq. (1), the proposed solving procedure is started by estimation of optimal solution for decision variable, $x$. This initial solution will be in an exploratory process till the new solution is achieved. The optimal solution for the lower level, i.e. $y^{*}$, is obtained in each iteration, and it returns to the higher level of the model. The process will go on till an optimal or near-optimal solution is found for the leader level of the model.

\subsection{Solving BLP using PSO}

As an evolutionary meta-heuristic algorithm, PSO has been organized based on an initial population and a random solution [29]. Each particle, i.e. a location vector, is generated randomly in the feasible area of the problem. Particles need velocity to achieve a better position. Thus, a velocity vector, which is usually zero, is generated for each particle. Then, positions of particles are updated in each iteration of the process, according to Eqs. (25) and (26). The velocity of each particle is calculated by Eq. (25), and then the position of a particle is updated by Eq. (26) according to previous position and velocity:

$$
V_{i}^{t+1}=W V_{i}^{t}+c_{1} r_{1}\left(p_{i}^{t}-x_{i}^{t}\right)+c_{2} r_{2}\left(p_{g}^{t}-x_{i}^{t}\right),
$$

$$
x_{i}^{t+1}=x_{i}^{t}+V_{i}^{t+1}
$$

where $i$ is used for particles' indexing; $t$ is a iteration counter; $x_{i}^{t}$ and $V_{i}^{t}$ indicate position and velocity of particle $i$ in $t$ iteration, respectively; $p_{i}$ is the best position found for the particle $i$ so far and $p_{g}$ is the best position obtained for all particles so far; $c_{1}$ and $c_{2}$ are constants; $r_{1}$ and $r_{2}$ are random numbers generated in the range of $0-1$; and $W$ is the weight inertia to control diversification and intensification of the problem space. $W$ is adjusted to each iteration using Eq. (27):

$$
W=W_{\min }+\left(W_{\max }-W_{\min }\right) .\left(\frac{\text { iter }_{\max }-\text { iter }}{\text { iter }_{\max }}\right) .
$$

$W_{\max }$ and $W_{\min }$ are the initial and final values of their inertia weight; iter $_{\max }$ is the iteration maximum and iter is the current iteration.

\subsubsection{The proposed PSO algorithm: Buyer as a leader}

Pseudocode of the formulated BLP is shown in Algorithm 1 considering the buyer as a leader. The algorithm is started by the initial data and the proper values for needing parameters. The initial solutions are generated based on the swarm-sized particles. The

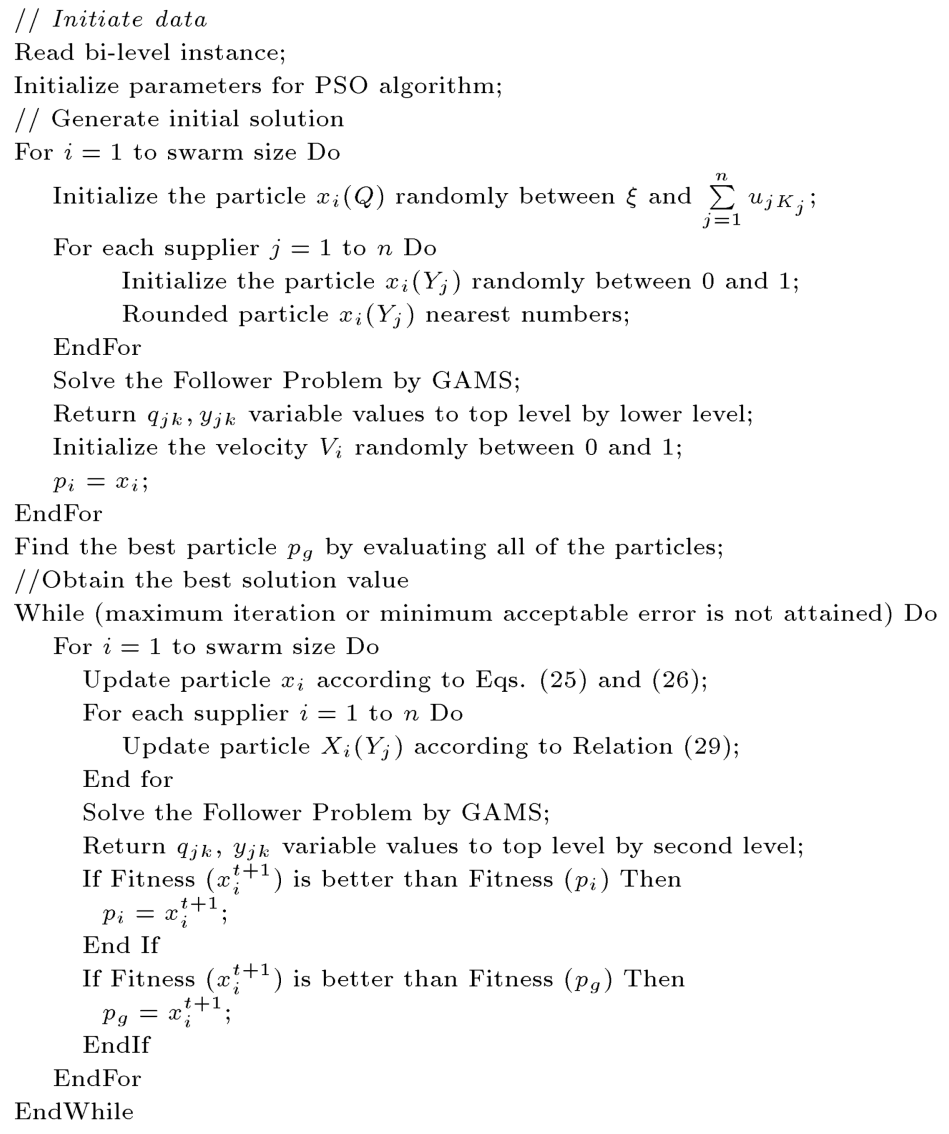

Algorithm 1. The proposed PSO algorithm: Buyer as a leader. 
encoding scheme of solution vectors is displayed in Eq. (28):

$$
x=\left[Q, Y_{1}, Y_{2}, \cdots, Y_{n}\right] .
$$

$Q$ is generated within the range of $\left[\xi, \sum_{j=1}^{n} u_{j K_{j}}\right]$ randomly, and also a random variable in the range of $0-1$ is generated for quantity, $Y_{j}$. If the random value is less than 0.5 , then $Y_{j}$ equals zero, unless it equals one. For each candidate solution, a fitness function is introduced to balance the solution fitness and optimization purpose. As also shown in Figure 1, for each given $x$, the optimal solution to the follower problem is obtained as $y^{*}$. It is possible to obtain the fitness values for each solution by Eq. (3).

The generated solutions of the leader are presented to the follower. The follower problem is solved, and then the values of $q_{j k}$ and $y_{j k}$ are returned to the leader to calculate the objective function. The initial velocity of every particle is randomly determined in the range $\left[\begin{array}{ll}0 & 1]\end{array}\right.$. The best position of each particle in each phase is obtained by $p_{i}$. Initially, $p_{i}$ for each particle is equal to the initial position of each particle. After calculating the objective value of all particles, the best position for $p_{g}$ is achieved using the best objective function.

After generating the initial population, it is possible to improve efficiency and performance of the particles. Using Eqs. (25) and (26), the new values of the velocity and position for all particles are obtained at each step of iteration. Also, $Y_{j}$ is binary variable in each iteration which can be updated by Eq. (29):

$$
x_{i}^{t+1}=\left\{\begin{array}{ll}
0 & \text { if } x_{i}^{t+1}<0.5 \\
1 & \text { otherwise }
\end{array}\right\} .
$$

Given the previous and current values of the objective function, the best position is obtained for every particle. If the new answer is better than the previous one, the position of the particle is replaced with a new the position, which means to change $p_{i}$. Additionally, $p_{g}$ is replaced with new values if the other particles contain better objective functions. Finally, after obtaining the maximum iteration or the closest distance from solutions, the defined error algorithm is terminated, and the best answer will be achieved. The proposed PSO structure is discussed in the next section regarding the vendor as a leader.

\subsubsection{The proposed PSO algorithm: Vendor as a leader}

Bi-level PSO pseudocode, considering vendor as a leader, is illustrated in Algorithm 2. The encoding scheme of solution vectors is displayed in Eq. (30):

$$
x=[\underbrace{q_{11}, \cdots, q_{1 K_{1}}}_{Q_{1}}, \cdots, \underbrace{q_{n 1}, \cdots, q_{n K_{n}}}_{Q_{n}}] .
$$

First, supplier 1 is chosen by the vendor and a random number is generated between zero and one. The first supplier is omitted if the generated number is less than 0.5. Otherwise, the supplier is selected. The other random integer number is generated between 1 and $K_{j}$ to randomly select a discount interval. Finally, an amount of order is randomly generated according to the selected supplier. This procedure is repeated for all suppliers. If none of the suppliers is selected, this procedure is repeated from the beginning.

After generating initial data, the generated solutions, obtained by the leader, are handed in to the follower. The problem of the follower is solved using GAMS software, and the leader is provided with $Q, Y_{j}$ values to calculate the objective value. After this step, the algorithm goes to improve the quality of the solution. Solutions are used to get new values at each step of iteration for all particles in Eqs. (25) and (26). The local search, introduced by Kamali et al. [30], is used to improve the updated solutions. Given the two suppliers indexed by $i$ and $j$, in the first phase, the number of the suppliers' orders is exchanged. The replacement is carried out if they are interchangeable considering the suppliers capacities introduced by $u_{i k_{i}}, u_{j k_{j}}, i, j=$ $1, \cdots n, k_{i}=1, \cdots, k_{i}$, and $k_{j}=1, \cdots, k_{j}$. If the cost of the resulted solution is lower than that of the previous one, the solution is replaced; otherwise, it is discarded. This procedure is done for all elements of the paired suppliers. Given the first supplier, in the next phase, its order quantity is added to the other supplier who has an extra necessary capacity. If the cost of the new solution is lower, the order quantity is assigned to the best supplier. As it is done in the first phase, this procedure is repeated for all elements of the paired suppliers. Results of calculations and sensitivity analysis of an example will be discussed in the next section.

\section{Computational results}

The required codes for solving the programs are written by MATLAB software, version 7.10.0 in Windows XP (Intel Core 2 Duo CPU 2.83 GHz, 2 GB RAM).

\subsection{Numerical example}

A numerical example is offered to confirm the validity of the formulated BLP models and the proposed solution procedures. Suppose a SC involving a buyer and a vendor with four suppliers. First, the buyer wants to know which of four suppliers can be selected with its order quantity per period. The annual demand of the buyer is 100,000 units and the buyer should satisfy the demand. Suppliers offer products with all-unit quantity discount and discount intervals, illustrated in Table 1. The other information required by suppliers is shown in Table 2. Inventory holding rate per unit is 2.6 in the buyer's level. 


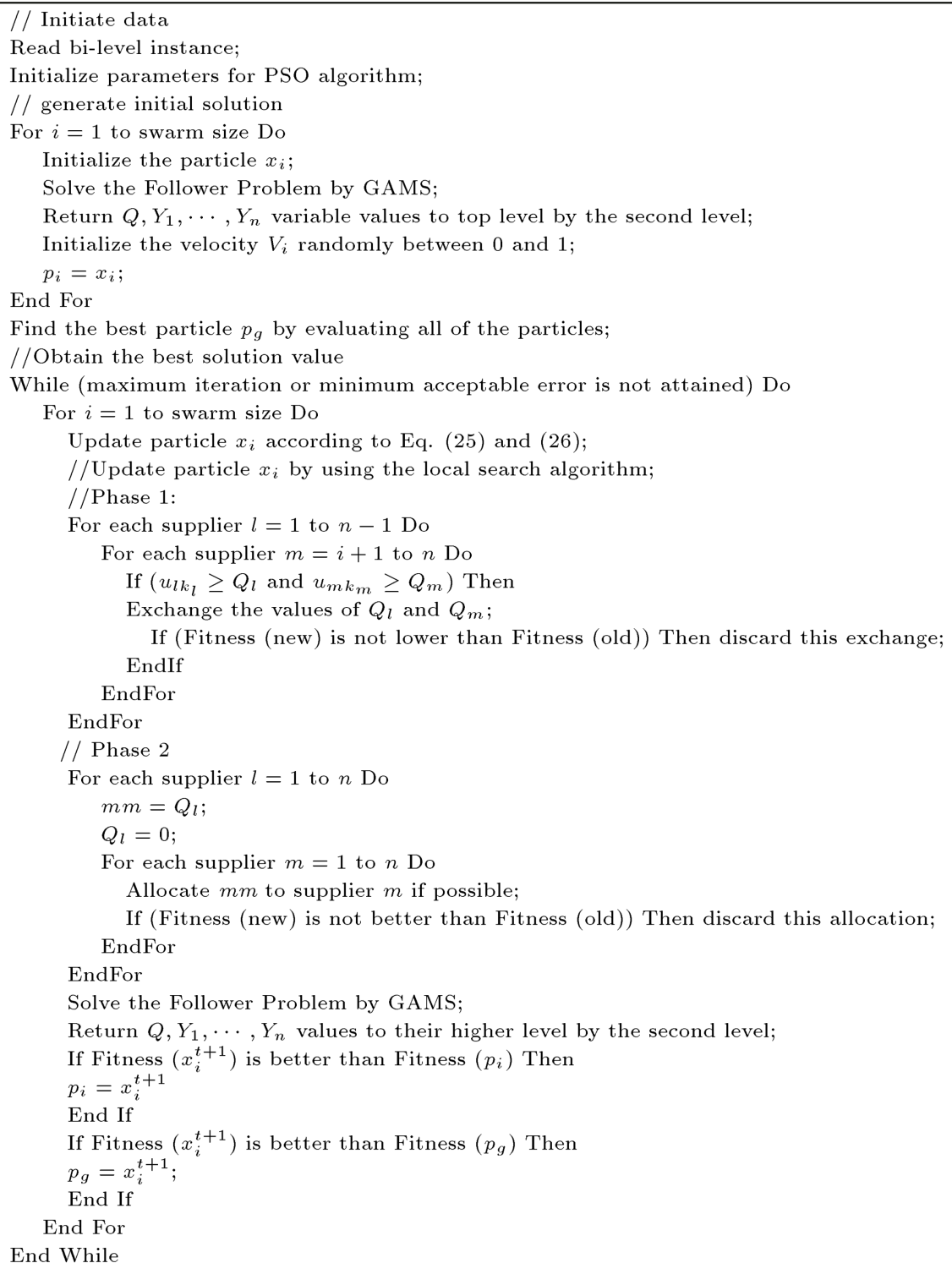

Algorithm 2. Proposed PSO algorithm: Vendor as a leader.

Table 1. Numerical example of the discount intervals.

\begin{tabular}{|c|c|c|c|c|c|c|c|c|c|}
\hline Supplier & \multicolumn{3}{|c|}{ Intervals } & Unit price & Supplier & \multicolumn{3}{|c|}{ Intervals } & Unit price \\
\hline \multirow{6}{*}{1} & 0 & $<Q<$ & 5000 & 9.0 & \multirow{6}{*}{2} & 0 & $<Q<$ & 2000 & 9.1 \\
\hline & 5000 & $\leq Q<$ & 10000 & 8.9 & & 2000 & $\leq Q<$ & 4000 & 9.0 \\
\hline & 10000 & $\leq Q<$ & 15000 & 8.8 & & 4000 & $\leq Q<$ & 6000 & 8.9 \\
\hline & 15000 & $\leq Q<$ & 20000 & 8.7 & & 6000 & $\leq Q<$ & 8000 & 8.8 \\
\hline & 20000 & $\leq Q<$ & 25000 & 8.6 & & 8000 & $\leq Q<$ & 10000 & 8.7 \\
\hline & 25000 & $\leq Q<$ & 30000 & 8.5 & & 10000 & $\leq Q \leq$ & 20000 & 8.6 \\
\hline \multirow{4}{*}{3} & 0 & $<Q<$ & 3000 & 8.7 & \multirow{4}{*}{4} & 0 & $<Q<$ & 4000 & 10.5 \\
\hline & 3000 & $\leq Q<$ & 6000 & 8.6 & & 4000 & $\leq Q<$ & 8000 & 10.4 \\
\hline & 6000 & $\leq Q<$ & 9000 & 8.5 & & 8000 & $\leq Q<$ & 12000 & 10.3 \\
\hline & 9000 & $\leq Q<$ & 12000 & 8.4 & & 12000 & $\leq Q<$ & 16000 & 10.2 \\
\hline
\end{tabular}


Table 2. Suppliers information.

\begin{tabular}{cccccc}
\hline Supplier & $\boldsymbol{z}$ & $\boldsymbol{S}$ & $\boldsymbol{P}$ & $\boldsymbol{A}$ & $\boldsymbol{h}$ \\
\hline 1 & 4.04 & 43 & 35108 & 40 & 2.29 \\
2 & 6.48 & 39 & 29898 & 19 & 1.96 \\
3 & 7.17 & 42 & 35785 & 25 & 2.74 \\
4 & 5.87 & 30 & 68777 & 39 & 0.54 \\
\hline
\end{tabular}

Table 3. Suggested PSO parameters.

\begin{tabular}{cc}
\hline Parameter & Quantity \\
\hline Swarm size & 100 \\
$W_{\max }$ & 0.7 \\
$W_{\min }$ & 0.2 \\
$c_{1}$ & 0.8 \\
$c_{2}$ & 0.8 \\
\hline
\end{tabular}

The values of the proposed PSO parameters are shown in Table 3 . These values have been obtained through trial and error.

The results are shown in Table 4, i.e. objective function values for higher and lower levels. Also, the information in this table shows the order quantities of the four suppliers. The results show the fact that the sum of cost for the integrated approach is lower than the two bi-level models. However, the individual cost of vendor or buyer for the bi-level strategies is lower than that of the integrated model. For the buyer as a leader, costs are reduced by $3 \%$ (from 890,717 to 865,286 ), and for the vendor as a leader, they are reduced by $12 \%$ (from 597,906 to 526,822 ). The table also includes solutions to the integrated model. The objective function value of the integrated approach is the sum of the total costs of the buyer and vendor. But, compared with bi-level approaches, these values are also included separately. Although the sum of the costs in the integrated approach is lower than that of the bi-level approach, it should be noted that the higher level as the prior interest resulted in a much higher level. The objective function value for the leaders is decreasing in the bi-level model. The impact of changing of parameters on the obtained results is discussed in the next section.

\subsection{Sensitivity analysis}

Sensitivity analysis is done by changing the parameters in the range of 10 to $50 \%$ and by changing one of the parameters and fixing the other parameters with the initial values. The results are shown in Tables 5 and 6. Results in Table 5 show that when the buyer plays the role of the leader, changes in the objective function are highly sensitive to changes in the value of demand, but less sensitive to those of production rates. Also, changes in the objective function show

Table 5. Sensitivity analysis for the buyer as leader.

\begin{tabular}{ccccccc}
\hline \multirow{2}{*}{ Parameters } & \multicolumn{6}{c}{ Changes in parameter values (\%) } \\
\cline { 2 - 7 } & -50 & -30 & -10 & +10 & +30 & +50 \\
\hline$D$ & -49 & -30 & -10 & +24 & +43 & +64 \\
$h_{b}$ & -2 & -1 & 0 & 0 & +1 & +1 \\
$z_{i}$ & 0 & 0 & 0 & 0 & 0 & 0 \\
$S_{i}$ & 0 & 0 & 0 & 0 & 0 & 0 \\
$P_{i}$ & Impossible & +11 & +13 & 0 & 0 & -1 \\
$A_{i}$ & 0 & 0 & 0 & 0 & 0 & 0 \\
$h_{i}$ & 0 & 0 & 0 & 0 & 0 & 0 \\
\hline
\end{tabular}

Table 6. Sensitivity analysis for the vendor as leader.

\begin{tabular}{ccccccc}
\hline \multirow{2}{*}{ Parameters } & \multicolumn{6}{c}{ Changes in parameter values (\%) } \\
\cline { 2 - 7 } & -50 & -30 & -10 & +10 & +30 & +50 \\
\hline$D$ & -56 & -34 & -11 & +12 & +37 & +64 \\
$h_{b}$ & 0 & 0 & 0 & 0 & 0 & 0 \\
$z_{i}$ & -50 & -30 & -10 & +10 & +30 & +50 \\
$S_{i}$ & 0 & 0 & 0 & 0 & 0 & 0 \\
$P_{i}$ & Impossible & +8 & +2 & -1 & -4 & -6 \\
$A_{i}$ & 0 & 0 & 0 & 0 & 0 & 0 \\
$h_{i}$ & 0 & 0 & 0 & 0 & 0 & 0 \\
\hline
\end{tabular}

Table 4. The results of the numerical example.

\begin{tabular}{lccc}
\hline & $\begin{array}{c}\text { The bi-level } \\
\text { approach assuming } \\
\text { the buyer as a leader }\end{array}$ & $\begin{array}{c}\text { The bi-level } \\
\text { approach assuming } \\
\text { the vender as a leader }\end{array}$ & $\begin{array}{c}\text { Integrated } \\
\text { approach } \\
\text { (Kamali et al.) }\end{array}$ [30] \\
\hline Order to supplier 1 & 21068.29 & 1259.63 & 3009.77 \\
Order to supplier 2 & 17941.66 & 0 & 2563.13 \\
Order to supplier 3 & 21000 & 0 & 3000.00 \\
Order to supplier 4 & 0 & 2328.25 & 0 \\
Buyer's objective function value & 865286 & 1002079 & 890717 \\
Vendor's objective function value & 656529 & 526822 & 597906 \\
The integrated objective function value & 1521815 & 1528901 & 1488623 \\
\hline
\end{tabular}


little sensitivity to those in the values of holding costs. Results in Table 6 indicate that when the vendor plays the role of the leader, changes in the objective function in relation to the quantity of the demand and variable costs are very sensitive. Changes in the values of other parameters have no specific effect on the objective function. $50 \%$ reduction in production rates' change in both cases is not possible because it will not satisfy customer's demand.

\section{Conclusion}

The order allocation problem with the supplier selection under an all-unit quantity discount policy with buyer as a leader was formulated and compared to the model with vendor as a leader in this paper. Two bilevel models were studied in a supply chain network design problem with a hierarchical structure. It was assumed, in this study, that both buyer and vendor play the leadership role in a BLP model structure. The latter reacts to the decisions made by the leader. Since NBLP problems were considered as a NP-hard problem, PSO algorithm was proposed to solve NBLP suggested nonlinear models. Using the proposed solved algorithms, an example was compared with solutions from the integrated model. The sensitivity analysis was conducted to assess the changes in the values of the parameters. When one of the players, leader or follower, has dominant power in the supply chain, the proposed models can be used to choose suppliers in a supply chain. The results can be used as a management managerial insight to design strategic models of bilevel inventory. The impact of demand on uncertainty can be studied as a further research in this area. It is also possible to study the problem of selecting the suppliers from multi-objective views. Another area of study is to assess the models in multi products and alternative product conditions. The study also considered suppliers in the form of a single company, while they can be studied as an independent one or a company in a competitive environment.

\section{References}

1. Golpîra, H., Zandieh, M., Najafi, E. and Sadi-Nezhad, S. "A multi-objective multi-echelon green supply chain network design problem with risk-averse retailers in an uncertain environment", Scientia Iranica, Transaction E, Industrial Engineering, 24(1), p. 413 (2017).

2. Huang, Y., Huang, G.Q. and Newman, S.T. "Coordinating pricing and inventory decisions in a multi-level supply chain: A game-theoretic approach", Transportation Research Part E: Logistics and Transportation Review, 47(2), pp. 115-129 (2011).

3. Calvete, H.I., Galé, C. and Oliveros, M.J. "Bilevel model for production-distribution planning solved by using ant colony optimization", Computers \& Operations Research, 38(1), pp. 320-327 (2011).

4. Calvete, H.I. and Galé, C. "On linear bilevel problems with multiple objectives at the lower level", Omega, 39(1), pp. 33-40 (2011).

5. Angelo, J.S. and Barbosa, H.J. "A study on the use of heuristics to solve a bilevel programming problem", International Transactions in Operational Research, 22(5), pp. 861-882 (2015).

6. Gaski, J.F. "The theory of power and conflict in channels of distribution", The Journal of Marketing, 48(3), pp. 9-29 (1984).

7. Sinha, A., Malo, P., Frantsev, A. and Deb, K. "Multiobjective Stackelberg game between a regulating authority and a mining company: A case study in environmental economics", In Evolutionary Computation (CEC), IEEE Congress, pp. 478-485 (2013).

8. Friedlander, A. and Gomes, F.A. "Solution of a truss topology bilevel programming problem by means of an inexact restoration method", Computational \& Applied Mathematics, 30(1), pp. 109-125 (2011).

9. Costantino, N., Dotoli, M., Falagario, M., Fanti, M.P., Mangini, A.M., Sciancalepore, F. and Ukovich, W. "A hierarchical optimization technique for the strategic design of distribution networks", Computers \& Industrial Engineering, 66(4), pp. 849-864 (2013).

10. Gao, Y., Zhang, G., Lu, J. and Wee, H.M. "Particle swarm optimization for bi-level pricing problems in supply chains", Journal of Global Optimization, 51(2), pp. $245-254$ (2011).

11. Hansen, P., Jaumard, B. and Savard, G. "New branchand-bound rules for linear bilevel programming", SIAM Journal on Scientific and Statistical Computing, 13(5), pp. 1194-1217 (1992).

12. Ma, W., Wang, M. and Zhu, X. "Hybrid particle swarm optimization and differential evolution algorithm for bi-level programming problem and its application to pricing and lot-sizing decisions", Journal of Intelligent Manufacturing, 26(3), pp. 471-483 (2015).

13. Pan, Q., An, Z. and Qi, H. "Exact penalty method for the nonlinear bilevel programming problem", Wuhan University Journal of Natural Sciences, 15(6), pp. 471475 (2010).

14. Roghanian, E., Aryanezhad, M.B. and Sadjadi, S.J. "Integrating goal programming, Kuhn-Tucker conditions, and penalty function approaches to solve linear bi-level programming problems", Applied Mathematics and Computation, 195(2), pp. 585-590 (2008).

15. Nishi, T., Hiranaka, Y. and Grossmann, I.E. "A bilevel decomposition algorithm for simultaneous production scheduling and conflict-free routing for automated guided vehicles", Computers \& Operations Research, 38(5), pp. 876-888 (2011). 
16. Li, M., Lin, D. and Wang, S. "Solving a type of biobjective bilevel programming problem using NSGA-II", Computers \& Mathematics with Applications, 59(2), pp. 706-715 (2010).

17. Angelo, J.S., Krempser, E. and Barbosa, H.J. "Differential evolution for bilevel programming", In Evolutionary Computation (CEC), IEEE Congress on, pp. 470-477 (June, 2013).

18. Deb, K., Gupta, S., Dutta, J. and Ranjan, B.” Solving dual problems using a coevolutionary optimization algorithm", Journal of Global Optimization, 57(3), pp. 891-933 (2013).

19. Yu, Y., Huang, G.Q. and Liang, L. "Stackelberg gametheoretic model for optimizing advertising, pricing and inventory policies in vendor managed inventory (VMI) production supply chains", Computers \& Industrial Engineering, 57(1), pp. 368-382 (2009).

20. Kis, T. and Kovács, A. "Exact solution approaches for bilevel lot-sizing", European Journal of Operational Research, 226(2), pp. 237-245 (2013).

21. Sadigh, A.N., Mozafari, M. and Karimi, B. "Manufacturer-retailer supply chain coordination: A bi-level programming approach", Advances in Engineering Software, 45(1), pp. 144-152 (2012).

22. Wee, H.M., Lee, M.C., Yang, P.C. and Chung, R.L. "Bi-level vendor-buyer strategies for a time-varying product price", Applied Mathematics and Computation, 219(18), pp. 9670-9680 (2013).

23. Verma, R. and Pullman, M.E. "An analysis of the supplier selection process", Omega, 26(6), pp. 739-750 (1998).

24. Rosenblatt, M.J., Herer, Y.T. and Hefter, I. "An acquisition policy for a single item multi-supplier system", Management Science, 44(1), pp. 96-100 (1988).

25. Xia, W. and Wu, Z. "Supplier selection with multiple criteria in volume discount environments", Omega, 35(5), pp. 494-504 (2007).

26. Zhang, J.L. and Zhang, M.Y. "Supplier selection and purchase problem with fixed cost and constrained order quantities under stochastic demand", International Journal of Production Economics, 129(1), pp. 1-7 (2011).

27. Mohamadi, M., Tezenji, F.R., Pasandideh, S.H.R. and Koupaei, M.N. "An integrated model for supplier location-selection \& order allocation under capacity constraints in an uncertain environment", Scientia Iranica Transaction E, Industrial Engineering, 23(6), p. 3009 (2016).

28. Neyestani, M.S., Jolai, F. and Golmakani, H.R. "An evolutionary algorithm for supplier order allocation with fuzzy parameters considering linear and volume discount", Scientia Iranica, Transactions E, Industrial Engineering, 22(3), pp. 1130-1141 (2015).
29. Eberhart, R. and Kennedy, J. "A new optimizer using particle swarm theory", The Sixth International Symposium on Micro Machine and Human Science, IEEE 43 (1995).

30. Kamali, A., Ghomi, S.F. and Jolai, F. "A multiobjective quantity discount and joint optimization model for coordination of a single-buyer multi-vendor supply chain", Computers \& Mathematics with Applications, 62(8), pp. 3251-3269 (2011).

\section{Biographies}

Fayegh Zaheri accomplished his BSc degree in Industrial Engineering at Amirkabir University of Technology, Tehran, Iran (1994), and MSc degree in Industrial Engineering at University of Tehran, Iran (2001). He has been working as a PhD candidate at Sciences \& Research Branch, Islamic Azad University, Tehran, Iran. His research interests are supply chain management, scheduling and soft computing.

Mostafa Zandieh accomplished his BSc degree in Industrial Engineering at Amirkabir University of Technology, Tehran, Iran (1994-1998), and MSc degree in Industrial Engineering at Sharif University of Technology, Tehran, Iran (1998-2000). He obtained his $\mathrm{PhD}$ degree in Industrial Engineering from Amirkabir University of Technology, Tehran, Iran (2000-2006). Currently, he is an Associate Professor at Industrial Management Department, Shahid Beheshti University, Tehran, Iran. His research interests are production planning and scheduling, financial engineering, quality engineering, applied operations research, simulation, and artificial intelligence techniques in the areas of manufacturing systems design.

Mohammad Taghi Taghavifard is an Associate Professor in the College of Management and Accounting at Allameh Tabataba'i University (Tehran-Iran). He received his MS degree in Management Science from University of Tennessee (Knoxville, TN-USA) and his $\mathrm{PhD}$ degree in Industrial Engineering from Wichita State University (Wichita, KS-USA) in 1993.

He has published numerous scientific articles in the fields of Industrial Engineering (IE) and Information Technology (IT) in several national and international refereed journals and in many conference proceedings. He is also the author of many books in various fields of IE and IT. His research interests include sequencing and scheduling techniques, the analysis and optimization of manufacturing, application of IT in management, applications of meta-heuristic methods, and applications of MCDM. He has served as a consultant to many private originations. 